\title{
Cornual Pregnancy with Uterine Rupture: A Case Report
}

\author{
(1) Vinodhini Elangovan, (1) Jen Heng Pek \\ Department of Emergency Medicine, Sengkang General Hospital, Sengkang, Singapore
}

\begin{abstract}
Cornual pregnancy is a rare and hazardous form of ectopic pregnancy that causes massive hemorrhage and death. Presented herein is a 38-year-old female patient who was brought to our Emergency Department at $30+6$ weeks of gestation for abdominal pain and hypotension due to uterine rupture, as a result of left cornual pregnancy. Here, the confusion of cornual pregnancy with normal intrauterine pregnancy and its associated catastrophic complications of uterine rupture and fetal demise are highlighted. This case report demonstrates the importance for an emergency physician to maintain the suspicion of ectopic pregnancy even at advanced gestational age.
\end{abstract}

Keywords: ectopic pregnancy, emergency, uterine rupture

\section{Introduction}

Ectopic pregnancy occurs in $1 \%$ to $2 \%$ of pregnancies when the embryo is implanted outside the endometrial cavity, with the most common site being the fallopian tube (1-3). Identified risk factors for ectopic pregnancy include tubal damage due to infection or prior surgery, in-vitro fertilization and smoking (1). Ectopic pregnancy is an important cause of maternal mortality in the first trimester, making it an important diagnosis to exclude. A confidential inquiry into maternal and child health from 2003 to 2005 has identified that 10 out of 295 maternal deaths were attributable to ectopic pregnancies (4).

Cornual pregnancy, a rare sub-type making up $2 \%$ to $4 \%$ of ectopic pregnancies, occurs in the interstitial portion of the fallopian tube and invades through the uterine wall $(5,6)$. This is the most hazardous form of ectopic pregnancies due to the diagnostic dilemmas associated with it (7). Uterine rupture may occur in up to $20 \%$ of the cases that progress beyond 12 weeks of gestational age, resulting in massive hemorrhage and high mortality rates (8). In this case report, we present a third trimester cornual pregnancy, which is a rare phenomenon, that resulted in a uterine rupture.

\section{Case Report}

A 38-year old Chinese female, with gestational diabetes and hypertension, was brought to the Emergency Department (ED) by Emergency Medical Services (EMS) for abdominal pain and hypotension. She was 30+6 weeks pregnant (Gravida 3, Parity 1). Her past obstetric history included a live birth delivered via lower segment Caesarean section due to non-reassuring fetal status 18 years ago, and a first trimester miscarriage 8 years ago. For her current pregnancy, she was receiving routine prenatal care. She had an episode of threatened miscarriage during the second trimester at 15+1 weeks for which she was treated with intramuscular hydroxyprogesterone and oral progesterone. She recovered well and subsequent antenatal blood and ultrasound investigations were assessed to be normal with no suspicion of ectopic pregnancy or fetal and placental abnormality.

The patient developed generalized abdominal pain prior to the ED presentation. There was no vaginal bleeding or discharge. She also complained of non-vertiginous giddiness worse with postural changes and shortness of breath. The patient denied fever, nausea, vomiting, constipation, diarrhea, urinary symptoms, chest pain and neurological deficits. In the ED, her vitals were: temperature of $36.5{ }^{\circ} \mathrm{C}$, heart rate of 135 beats per minute, 
respiratory rate of 26 breaths per minute, blood pressure of $110 / 89 \mathrm{mmHg}$, and oxygen saturation of $99 \%$ with supplemental oxygen at 2 liters per minute. She was pale, diaphoretic and lethargic. Her pulses were weak and thready. She had generalized tenderness with guarding and rebound over her abdomen. No blood or fluid was noted on vaginal examination and the cervix was closed. The rest of the examination was unremarkable. Point of care ultrasound was performed and showed large amount of intra-abdominal free fluid (Figure 1) and the fetal heart rate was 170 beats per minute. The point of care hemoglobin was 7.1 $\mathrm{g} / \mathrm{dL}$ and lactate was $6.8 \mathrm{mmol} / \mathrm{L}$. The patient's blood pressure dropped precipitously to $50 / 36 \mathrm{mmHg}$ and her heart rate went up to 141 beats per minute.

The clinical impression was profound shock secondary to uterine rupture. She was started on massive transfusion protocol and tranexamic acid was administered. Dexamethasone was also given. Urgent surgical and obstetric consults were obtained and she was brought to the emergency operating theatre for exploratory laparotomy.

During the operation, there was extensive hemoperitoneum and the fetus was seen in the abdominal cavity. The fetus was delivered immediately and handed to the neonatal team. On exploration of the uterus, the point of rupture was at the left cornual region where the placenta was attached to. Left salpingectomy was performed and the uterus was repaired with hemostasis achieved. Oxytocin was administered and the patient was subsequently admitted to the Intensive Care Unit.

After delivery, the neonate was pale, cyanotic, listless and not crying with an APGAR score of 0 . The birth weight was 1,810 grams. He had no respiratory effort or pulse. He was

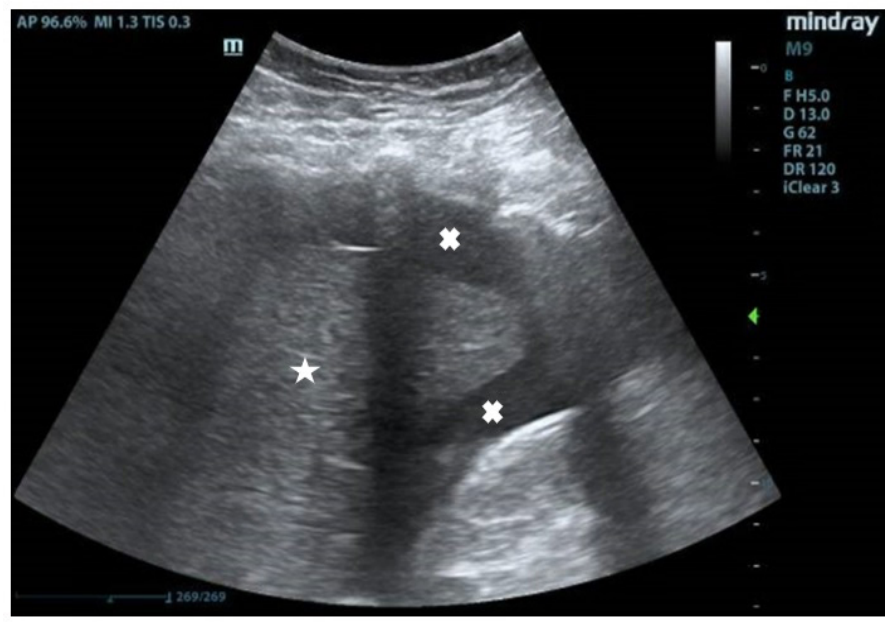

Figure 1. Point of Care Ultrasound Image. Ultrasound image of the hepatorenal space showing the presence of free fluid (hypoehoic areas indicated by white crosses) surrounding the liver (white star) warmed, intubated and chest compressions were initiated with administration of adrenaline and sodium bicarbonate, as well as boluses of normal saline and blood. The initial point of care $\mathrm{pH}$ was less than 6.5 with an unrecordable base excess, hemoglobin was $13.7 \mathrm{~g} / \mathrm{dL}$ and hypocount was $7.4 \mathrm{mmol} / \mathrm{L}$. After 93 minutes, the neonate experienced return of spontaneous circulation with the following vital signs: temperature of $31.2^{\circ} \mathrm{C}$, heart rate of 101 beats per minute, blood pressure of $48 / 16 \mathrm{mmHg}$, mean arterial pressure of $25 \mathrm{mmHg}$, oxygen saturation of $85 \%$ on ventilator (patient triggered ventilation mode, PIP 28, PEEP 5 and $\mathrm{FiO}_{2}$ $100 \%)$. He was started on dopamine infusion and brought to the neonatal intensive care unit. However, his condition continued to deteriorate and further resuscitation efforts were terminated due to poor prognosis and medical futility after discussion with the family. The cause of death was birth asphyxia. Bereavement support was provided to the family.

For the patient, her recovery was uneventful and she was discharged 5 days later. She was reviewed in the outpatient clinic one month later and remained well. She was advised against future pregnancy due to her increased risk of uterine rupture.

Consent was obtained from the patient for this case report.

\section{Discussion}

Uterine rupture can result in hemorrhagic shock, making it a true obstetric emergency with significant morbidity and mortality for the patient and the fetus. Therefore, an early diagnosis in the ED is crucial (9). However, as it is a rare condition with a reported incidence of $0.5 \%$ in all pregnancies, it is a diagnosis that is often missed (10). Uterine rupture can be divided into two main types (11). The first is a complete rupture involving the full thickness of the uterine wall and the second is an incomplete rupture when the visceral peritoneum remains intact. One key consideration in the evaluation of uterine rupture is to know whether or not the uterus is scarred. Uterine rupture more commonly occurs in a scarred uterus due to a previous lower segment Caesarean section, with a reported incidence between $0.22 \%$ to $0.5 \%(12,13)$. As maternal age advances, there is decreasing strength of the myometrium and defective healing of the uterine scar, along with increasing dysfunctional labor, the risk of uterine rupture is further increased (14). In the unscarred uterus, uterine rupture has been associated with trauma from obstetric maneuvers such as external cephalic version of breech to vertex presentation and assisted fundal pressure during delivery (11).

In this case report, the patient had advanced maternal age and previous lower segment Caesarean section, making the scar from previous lower segment Caesarean section the likely site of uterine rupture. However, at the time of operation, it was 
discovered that the point of rupture was at the left cornual region, away from the site of scar from previous lower segment Caesarean section. To date, there was only one case report of third trimester uterine rupture secondary to a cornual pregnancy (15). However, the patient described, sought prenatal care late in her pregnancy at 28 weeks. She was asymptomatic and a silent uterine rupture was picked up on initial ultrasound evaluation. This was in stark contrast to the patient in this case report as she had been followed up by an obstetrician closely throughout her pregnancy, and presented to the ED with abdominal pain and profound shock.

Cornual pregnancy poses a higher risk of rupture and hemorrhage compared to other types of ectopic pregnancies (16). However, it is difficult to diagnose with low ultrasonographic sensitivity and hence is commonly confused with normal intrauterine pregnancy $(16,17)$. Therefore, cornual pregnancy tends to present at 7 to 12 weeks of gestation, because of myometrial distensibility (5). A cornual pregnancy that continues beyond the second trimester, as in this case, is a rare phenomenon (15). A careful inspection of second trimester ultrasonogram can potentially prevent the rupture of a cornual pregnancy and its associated morbidity and mortality (18).

The use of point of care ultrasound greatly facilitated the diagnosis and management of this patient. Ultrasound is a safe imaging modality during pregnancy and its availability at the bedside in the ED made it a useful tool for emergency physicians in their assessment of patients who present with symptoms to determine the possibility of uterine rupture. Other than frank rupture as in this case, the applicability of point of care ultrasound can also be extended to patients who are not in need of immediate delivery and who present with very subtle symptoms (19). The diagnosis of uterine rupture is strongly suggested with the recognition of free fluid on point of care ultrasound as a result of blood in the peritoneal cavity (12). However, it may be difficult or impossible to visualize the actual site of rupture.

\section{Conclusion}

Cornual pregnancy is associated with fatal complications for the mother and fetus, necessitating the prompt diagnosis and management in the ED.

\section{Ethics}

Informed Consent: Consent was obtained from the patient for this case report.

Peer-review: Externally peer-reviewed.

\section{Authorship Contributions}

Surgical and Medical Practice: V.E., J.H.P., Concept: V.E., J.H.P., Design: V.E., J.H.P., Data Collection and/or Processing: V.E., J.H.P., Analysis and/or Interpretation: V.E., J.H.P., Literature Search: V.E., J.H.P., Writing: V.E., J.H.P.

Conflict of Interest: No conflict of interest was declared by the authors.

Financial Disclosure: The authors declared that this study received no financial support.

\section{References}

1. Elson CJ, Salim R, Potdar N, et al; on behalf of the Royal College of Obstetricians and Gynaecologists. Diagnosis and management of ectopic pregnancy. BJOG. 2016;123:e15-e55.

2. Bronson R. Ectopic pregnancy-still a challenge. Fertil Steril. 2018;110:1265-6.

3. Kulp JL, Barnhart KT. Ectopic pregnancy: diagnosis and management Womens Health (Lond). 2008;4:79-87.

4. Lewis G. The Confidential Enquiry into Maternal and Child Health (CEMACH). Saving Mothers' Lives: reviewing maternal deaths to make motherhood safer - 2003-2005. The Seventh Report on Confidential Enquiries into Maternal Deaths in the United Kingdom. London: CEMACH, 2007.

5. Shetty M, Shivananjaiah C, Swarup A, Sharma N, Sharma N. A Rare Case Report of Cornual Pregnancy. Obstet Gynecol Int J 2016;5:00179.

6. Chowdhury TS, Hasan H, Chowdhury T. Cornual pregnancy-an unusual site of pregnancy: a case report and literature review. BIRDEM Med J. 2020;10:64-7.

7. Faraj R, Steel M. Management of cornual (interstitial) pregnancy. Obstet Gynecol. 2007;9:249-55.

8. Soriano D, Vicus D, Mashiach R, Schiff E, Seidman D, Goldenberg M Laparoscopic treatment of cornual pregnancy: a series of 20 consecutive cases. Fertil Steril. 2008;90:839-43.

9. Berzon B, Gleenberg M, Offenbacher J, West D. Atypical presentation of hemorrhagic shock in pregnancy: a case highlighting the developing field of emergency medicine in Israel. BMC Emerg Med. 2019;19:70.

10. Motomura K, Ganchimeg T, Nagata C, Ota E, Vogel JP, Betran AP, et al. Incidence and outcomes of uterine rupture among women with prior caesarean section: WHO Multicountry Survey on Maternal and Newborn Health. Sci Rep. 2017;7:44093.

11. Turner MJ. Uterine rupture. Best Pract Res Clin Obstet Gynaecol. 2002;16:6979.

12. Bedi DG, Salmon A, Winsett MZ, Fagan CJ, Kumar R. Ruptured uterus: sonographic diagnosis. J Clin Ultrasound. 1986;14:529-33.

13. Smith D, Stringer E, Vladutiu CJ, Zink AH, Strauss R. Risk of uterine rupture among women attempting vaginal birth after cesarean with an unknown uterine scar. Am J Obstet Gynecol. 2015;213:80.e1-80.e5.

14. Hamilton EF, Bujold E, McNamara H, Gauthier R, Platt RW. Dystocia among women with symptomatic uterine rupture. Am J Obstet Gynecol. 2001;184:620-4.

15. Brewer H, Gefroh S, Munkarah A, Hawkins R, Redman ME. Asymptomatic uterine rupture of a cornual pregnancy in the third trimester: a case report. J Reprod Med. 2005;50:715-8.

16. Moawad NS, Mahajan ST, Moniz MH, Taylor SE, Hurd WW. Current diagnosis and treatment of interstitial pregnancy. Am J Obstet Gynecol. 2010;202:1529 
17. Tulandi T, Al-Jaroudi D. Interstitial pregnancy: results generated from the Society of Reproductive Surgeons Registry. Obstet Gynecol. 2004;103:47-50.

18. Hill AJ, Van Winden KR, Cook CR. A true cornual (interstitial) pregnancy resulting in a viable fetus. Obstet Gynecol. 2013;121(2 Pt 2 Suppl 1):427-30.
19. Wye D, Magotti R, Al-Mashat D, Benzie R, Condous G. Sonographic diagnosis of spontaneous uterine rupture at the site of cornual wedge resection scar - a case report. Australas J Ultrasound Med. 2014;17:45-8. 